\title{
A Time Pre-defined Variable Depth Search for Nurse Rostering
}

\author{
Edmund Burke ${ }^{1}$, Timothy Curtois ${ }^{1}$, Rong Qu ${ }^{1}$, Greet Vanden Berghe ${ }^{2}$ \\ ${ }^{1}$ School of Computer Science \& I.T., University of Nottingham, Jubilee Campus, \\ Wollaton Road, Nottingham. NG8 1BB. UK \\ ${ }^{2}$ Information Technology Engineering Department, KaHo St. Lieven, Gebr. \\ Desmetstraat 1, 9000 Gent. Belgium
}

\begin{abstract}
A variety of neighbourhood operators have been used in local search and metaheuristic approaches to solving nurse rostering problems. We test and analyse the efficiency of these neighbourhoods on benchmark problems taken from real world scenarios. A variable depth search is then developed based on the results of this investigation. The algorithm heuristically chains together moves and swaps which define the more effective search neighbourhoods. A number of heuristics for creating these chains were developed and we present experiments conducted to identify the best ones. As end users vary in how long they are willing to wait for solutions, a particular goal of this research was to create an algorithm that accepts a user specified computational time limit and uses it effectively. When compared against previously published approaches the results show that the algorithm is very competitive.
\end{abstract}

\section{Introduction}

High quality nurse rosters benefit nurses, patients and managers. Patients receive better healthcare if nurses are able to spend more time with them and mistakes are less likely if nurses are not stressed, tired and overworked due to poor scheduling and understaffing. Improved rosters can not only decrease nurse fatigue but also help them maximise the use of their leisure time and satisfy more of their personal requests. From a management point of view, better and more flexible scheduling can help retain nurses and aid recruitment, reduce tardiness and absenteeism, increase morale and productivity and provide better patient service and safety. Costs can also be reduced through having to hire less agency nurses to fill gaps in rosters and lower staff turnover.

Creating rosters, however, is a difficult and challenging search problem which requires the satisfaction of many constraints and the balancing of a variety of requirements. This time consuming and frustrating duty often falls to a head nurse who would rather be concentrating on their primary duty of caring for patients. Regular rescheduling may also be required to deal with staff sickness and absences. Computerised, automated rostering can remove the vast majority of this workload and provides a number of additional benefits including being able to create much higher quality schedules which are fair, impartial and satisfy more preferences. Legal requirements can also be checked, management statistics collected and monthly reports generated, all reducing paperwork. Other bonuses can range from linking to payroll systems to emailing out rosters or publishing them on the web once they are created.

Over the past 30-40 years, many different approaches have been used to solve nurse rostering problems of varying forms and complexity. Methods used include 
mathematical programming $[6,28,35,39,44,47]$, constraint programming $[20,36$, 38], goal programming and multi objective approaches [3, 4, 10, 27] and case-based reasoning $[7,8]$. A great variety of local search and metaheuristic approaches have also been applied to the problem, [2, 9, 12, 21, 37, 45] represent just a few recent examples. Many more can be found in the literature reviews of Burke et al. [17] and Ernst et al. [23]. There are, however, very few large-scale neighbourhood searches applied to nurse rostering problems.

As mentioned in a recent survey paper by Ahuja et al. [1] many successful very-large scale neighbourhood search techniques have appeared in various forms in the field of operations research. They commented, for example, that the well known LinKernighan algorithm for the travelling salesman problem can be viewed as a very large-scale neighbourhood search technique. Ahuja et al. categorised very large-scale neighbourhood methods into three similar classes, one of which are variable depth methods. Variable depth searches (including some ejection chain methods [24]) have been effectively applied to a number of optimisation problems, for example the vehicle routing problem [42] and the generalised assignment problem [48]. Many more examples of successful very-large scale neighbourhood searches can be found in the survey paper of Ahuja et al.

One paper that does introduce the application of such techniques to nurse rostering is that of Dowsland [22]. In her approach to providing an automated nurse rostering system, a tabu search is used that oscillates between decreasing cover violation and increasing roster quality. In each of these phases, two types of ejection chains are used. The first consists of a sequence of on/off day swaps between nurses and the other is made up of sequences of swapping week long work patterns between nurses. The chains are able to escape from bad optima that single on/off day or pattern swaps would not be able to escape from.

Another example of using such techniques in solving a nurse rostering problem is the method of Louw et al. [34] who also use an ejection chain approach. The compound move used is similar to Dowsland's chain of on/off day swaps. They noted "the compound move was able to achieve far superior reductions in the objective function value when compared to any of the elementary move types".

Very large-scale neighbourhood searches face the problem of exploring an exponentially large neighbourhood. Therefore, the key to developing effective ones is identifying heuristics and other mechanisms which can efficiently narrow or direct the search. This paper presents a variable depth search for nurse rostering and describes the heuristics and other features that make it successful.

In developing this algorithm, we have also created some test instances based on real world problems which are now publicly available and which are intended to become benchmark problems in this area. As has been mentioned in bibliographic surveys of nurse rostering problems, there is a great lack of test problems and benchmarks [17] and, as such, there is little comparative analysis of nurse rostering algorithms in the literature. Such analysis is very important to underpin scientific progress in this area. Although there are understandable reasons for this lack of publicly available problems (for example, there is no such thing as a typical nurse rostering problem and there is often an issue over the confidentiality of data) we hope our presentation of these problems goes some way to starting to fill this void.

In Section 2, the problem is introduced. Section 3 investigates various search neighbourhoods that have been used to solve nurse rostering problems. Section 4 presents the variable depth search and Section 5 contains the results from a number of 
experiments using this algorithm. Finally, Sections 6 and 7 discuss conclusions and future work respectively.

\section{Problem Description}

The data for this problem is based on and is similar to the data used in $[12,15,16]$. The algorithms previously developed for this problem were developed as part of a commercial system and as the data is from a real world environment it has been anonymized and any confidential information removed.

The problem requires the production of non-cyclical schedules which satisfy all hard constraints and as many working preferences and requests as possible. There are so many conflicting constraints and requests that if they were all hard constraints, a feasible solution would generally not exist. Instead, they are modelled as soft constraints and given relative priorities using weights.

In order to provide these data sets as benchmarks, we have made software implementations of these evaluation functions publicly available (http://www.cs.nott.ac.uk/ tec/NRP/). They can be used as a standard and ensure the accuracy of any new results. As well as making these evaluation functions available, we have also provided the application programming interface developed in order to create and test the algorithms we are presenting here. For example, it is possible to download the software for parsing and using the data, displaying, analysing and manually altering solutions and the algorithms themselves.

\subsection{Hard Constraints}

In a final roster or solution, if all hard constraints are not satisfied then the roster is not feasible and is thus not acceptable. There are three hard constraints:

1. A nurse cannot be assigned more than one of the same shift type per day.

2. Shifts which require certain skills can only be covered by (i.e. assigned to) nurses who have those skills.

3. The shift coverage requirements must be satisfied. For example, if a certain day requires three night shifts then there must be three employees present at that time to work during that shift. Over coverage is not permitted.

\subsection{Soft Constraints}

All other rules are modelled as soft constraints. Ideally these constraints should be satisfied but it will almost always be necessary to violate some of them in order to provide a feasible solution. When a soft constraint is broken, a penalty is incurred which is proportional to the importance of the constraint and the severity of its violation. The importance of each constraint is set using weights which are usermodifiable integer values. The higher the weight, the more important the constraint is relative to the other constraints.

With permission each employee can specify which constraints they wish to see imposed on their work schedule and also the specific parameters associated with that constraint. For example, one nurse may request not to work more than five consecutive days whereas another may wish to work no more than four consecutive days. Alternatively, some nurses may have part time contracts which stipulate less work than full time employees and so on. The large number and variety of rules which 
can be imposed makes it a very flexible system which can be used in many different environments. It also makes it possible to create problem instances which vary significantly in size and complexity. Therefore a problem solver which is robust and effective over a range of instances is paramount.

Some of the constraints which are considered include:

- Maximum number of shifts worked during the scheduling period.

- Maximum and minimum number of hours worked during the scheduling period or per week.

- Maximum and minimum number of consecutive working days.

- Maximum and minimum number of consecutive non-working days.

- Maximum number of a specific shift type worked. For example, maximum zero night shifts for the planning period or a maximum of seven early shifts. This constraint can also be specified for each week. For example, a nurse may request no late shifts for a certain week.

- Maximum number of weekends worked in four weeks (a weekend definition is also a user definable parameter i.e. Friday and/or Monday may be considered as part of the weekend).

- Maximum number of consecutive weekends worked.

- No night shifts before a weekend off.

- No split weekends, i.e. shifts on all days of the weekend or no shifts over the weekend.

- Identical shift types over a weekend. For example, if a nurse has a day shift on Saturday then he/she may prefer to have a day shift on Sunday also.

- Minimum number of days off after night shifts.

- Valid numbers of consecutive shift types. For example, three or four consecutive early shifts may be valid but two or five consecutive early shifts may not.

- Shift type successions. For example, if shift rotation is allowed, is shift type A allowed to follow B the next day?

- Maximum total number of assignments for all Mondays, Tuesdays, Wednesdays... For example, a nurse may request not to work on Wednesdays or may require to work a maximum of two Tuesdays during the scheduling period.

- Avoid a secondary skill being used by a nurse. Sometimes a nurse may be able to cover a shift which requires a specific skill but they may be reluctant to do so as it is not their preferred duty. An example would be a head nurse not wanting to stand in for a regular nurse.

The precise definition and implementation of these constraints can be found in [46].

The evaluation function used to determine the quality of a roster is the sum of all penalties calculated for each soft constraint violation in each nurse's individual schedule. The problem objective is to find a roster with as low a penalty as possible. Of course, due to the often conflicting and large number of constraints there is rarely a perfect roster with penalty zero. Instead, we can think of this as an optimisation problem with the goal of minimizing the roster's overall penalty.

Let: 
penalty $_{r}=$ the penalty for roster $r$.

penalty $_{r, n}=$ the penalty for the schedule of nurse $n$ in roster $r$.

$N_{r}=$ the number of nurses in roster $r$.

penalty $_{r}=\sum_{n=1}^{N_{r}}$ penalty $_{r, n}$

The objective is to minimize penalty $r$ for a given scheduling period with a fixed set of employees, cover requirements and a specific set of soft constraints and weights.

The algorithms presented in this paper are tested on ten instances which vary in the number of nurses, cover requirements, shift types, constraint types and priorities, personal requests and planning horizon. Table 1 provides some more information on the instances.

\begin{tabular}{lcccc}
\hline Instance & Nurses & \multicolumn{2}{c}{ Shift types } & \multicolumn{2}{c}{ Skill levels } & Planning horizon \\
\hline BCV-1.8.1 & 8 & 4 & 2 & 28 days \\
BCV-2.46.1 & 46 & 4 & 1 & 28 days \\
BCV-3.46.1 & 46 & 3 & 1 & 26 days \\
BCV-4.13.1 & 13 & 4 & 2 & 29 days \\
BCV-5.4.1 & 4 & 4 & 1 & 28 days \\
BCV-6.13.1 & 13 & 4 & 2 & 30 days \\
BCV-7.10.1 & 10 & 6 & 1 & 28 days \\
BCV-8.13.1 & 13 & 4 & 2 & 28 days \\
BCV-A.12.1 & 12 & 4 & 2 & 31 days \\
ORTEC01 & 16 & 4 & 1 & 31 days \\
\hline
\end{tabular}

Table 1 Problem Instances

Data sets one to eight are all based on real world data. Data set BCV-A.12.1 is a fictional test problem that uses all the possible constraint types available and contains many conflicting requests. ORTEC01 is instance 12 for the problem presented in [14] (the instance which the extra tests were performed on and the one commonly used by ORTEC). To model the problem with this system, some of its hard constraints have been changed to soft constraints with weights of 10,000. Therefore all solutions with penalty below 10,000 are feasible solutions to the original problem (solutions with penalties above 10,000 are not necessarily infeasible for the original problem though. All these instances and the best known solutions are available at http://www.cs.nott.ac.uk/ tec/NRP/.

\section{Search Neighbourhoods for Nurse Rostering}

This section describes two types of search neighbourhood that have been used to solve nurse rostering problems. Their applicability to the benchmark instances are then investigated. The results from this preliminary investigation are relevant to the variable depth search presented in Section 4.

\subsection{The Single Shift Neighbourhood}

Included in this category are all neighbourhoods that are identified by moves or swaps that change the assignment of up to two shifts, days on/off or variables at a time. Depending on the type of cover constraints (are there minimum and/or maximum shift 
cover requirements and are they hard or soft constraints?), these moves may involve either one nurse (e.g. [5, 10, 21]) or two nurses (e.g. [15, 16, 19, 27, 31, 37, 41]).

In the benchmark problems, the shift cover requirements are hard constraints and neither over nor under coverage are permitted. Therefore, once an initial feasible roster is constructed, only swaps or moves between two nurses are allowed. This ensures that the coverage constraint is not violated. Examples of these swaps are illustrated in Figure 1 and Figure 2. Figure 1, shows a section of a roster where L, N, $\mathrm{D}$ and $\mathrm{DH}$ are shifts and $\mathrm{G}, \mathrm{H}$ and $\mathrm{A}$ are nurses. Move $a$ involves the swapping of shift $\mathrm{L}$ and shift $\mathrm{N}$ on the 5 th of December between nurses $\mathrm{G}$ and $\mathrm{H}$ to give the resulting roster on the right. In Figure 2, move $b$ involves the assigning of shift $\mathrm{L}$ to nurse $\mathrm{G}$ from nurse $\mathrm{H}$ on 5 th December. This could be alternatively phrased as "swapping shift $\mathrm{L}$ and an empty shift between nurses $\mathrm{G}$ and $\mathrm{H}$ on the 5th of December."

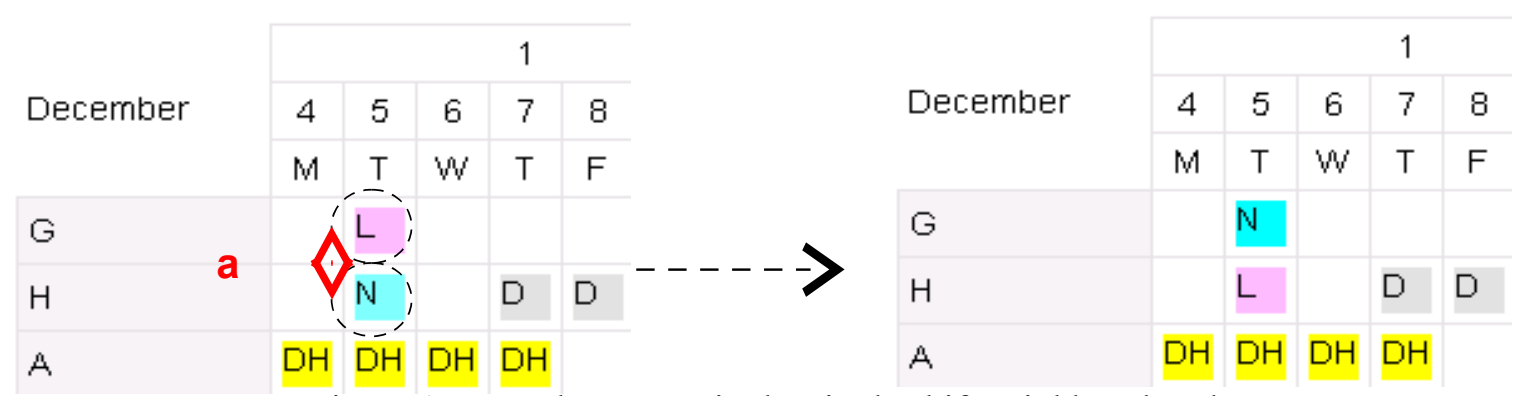

Figure 1 Example move a in the single shift neighbourhood

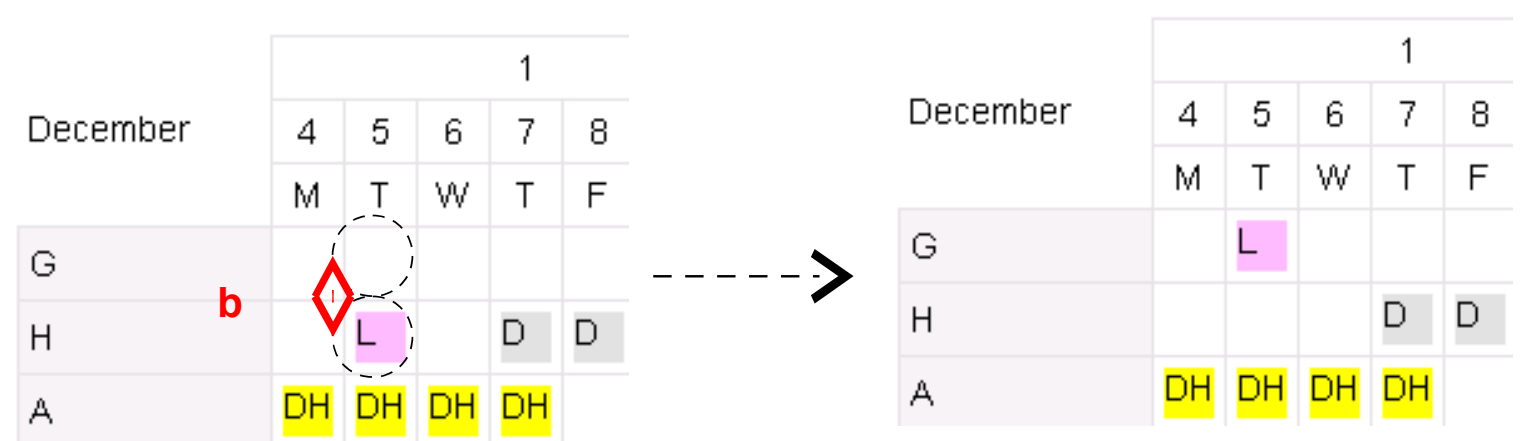

Figure 2 Example move $b$ in the single shift neighbourhood

The single shift neighbourhood, is the most commonly used neighbourhood in solving nurse rostering problems. It is a relatively small neighbourhood and easy to implement in a search algorithm. Even for the largest instances examined, using today's average desktop computer, this neighbourhood can be exhaustively searched and a local optimum can be reached quickly using a hill climber in this neighbourhood. Rosters produced using this approach, however, are not always of satisfactory quality and can usually be improved by an experienced human scheduler. Therefore, this neighbourhood is often, either incorporated into a more sophisticated method such as a metaheuristic and/or replaced with a larger neighbourhood.

\subsection{The Block Neighbourhood}

The single shift neighbourhood, on its own, is often not effective enough. Meyer auf'm Hofe [38] highlights its weakness with a specific example in which this 
neighbourhood, even if combined with a tabu list, would be unlikely to remove a particular violation as it requires the simultaneous change of eight (and only these eight) specific variables.

This section describes a larger neighbourhood defined by moves which would have been able to repair that particular violation. The neighbourhoods have recently been incorporated into search algorithms for nurse rostering and that approach has been shown to be very effective.

Included in this category of neighbourhoods are those defined by the swapping of all assignments on two or more adjacent days between two nurses. Examples of these swaps can be seen in Figure 3 and Figure 4. Figure 3 illustrates a move involving the swapping of a block of two adjacent days. On the 5th and 6th December, the $\mathrm{N}$ shifts of nurse $A$ are assigned to nurse $G$ and the $L$ shifts of nurse $G$ are assigned to nurse $A$. Here the block size is two as it involves two adjacent days. Figure 4 shows a move involving the swapping of a block of adjacent days of length three. Note that on 6th December, nurse A had no shift but this is still labelled as a swap with a block of days of length three.

As the block neighbourhood is a larger neighbourhood, its practical use was previously restricted by computational limitations. However, the recent dramatic increases in computing power have made a more aggressive use of this neighbourhood much more viable, as will be shown.

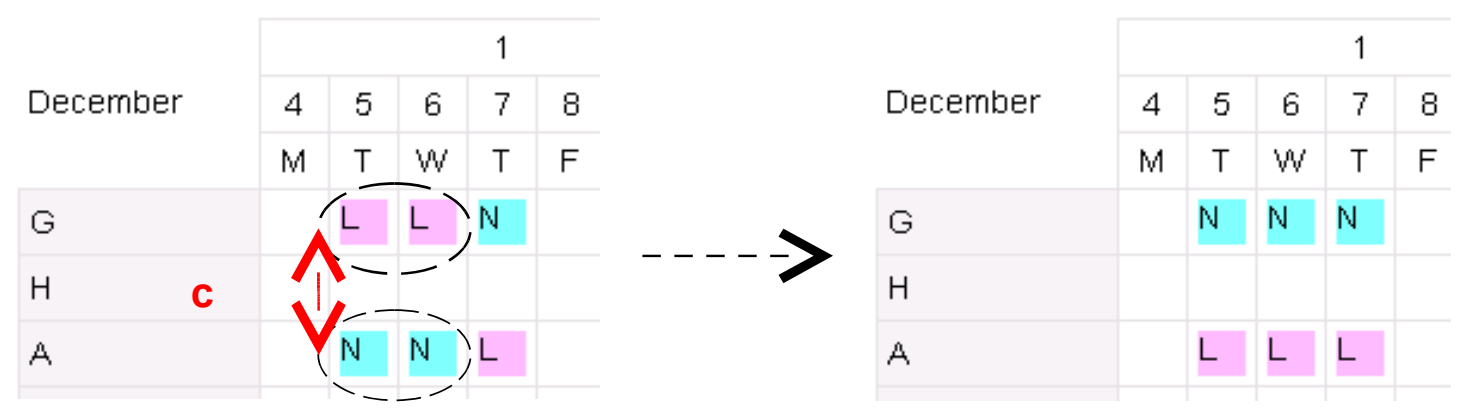

Figure 3 Example move $c$ in the block neighbourhood

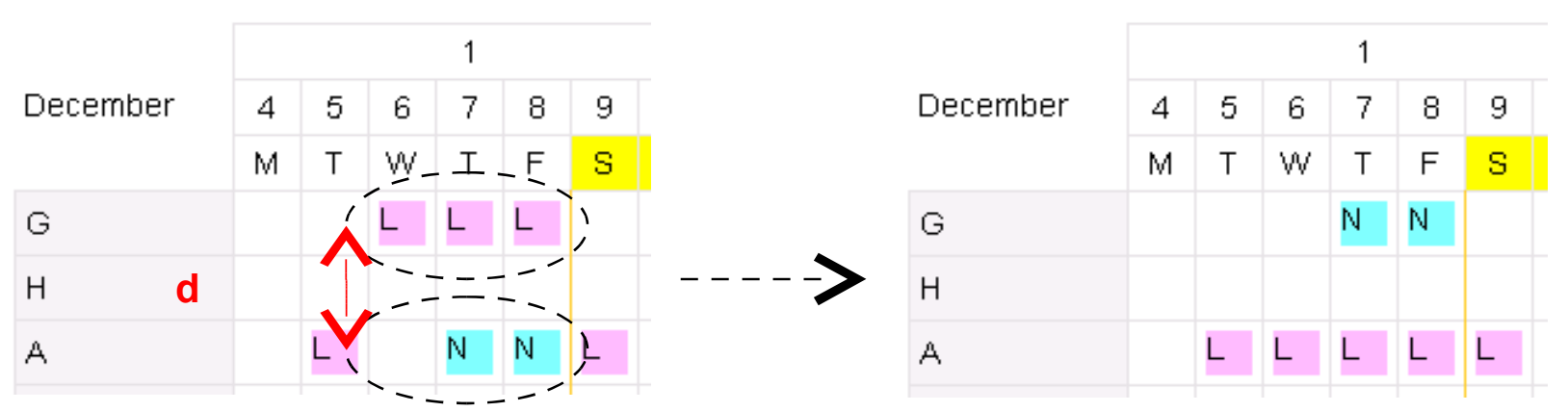

Figure 4 Example move $d$ in the block neighbourhood

An early example of the use of changing blocks of shifts can be found in [29], where, although a block neighbourhood is not actually used, varying size blocks of shifts are assigned to nurses to create initial rosters. In [26], Jan et al. swap assignments on blocks of days between nurses as a mutation operator (called escape) in a genetic algorithm but again do not use it as a search neighbourhood. The first use of this type 
of swap in a search neighbourhood can be found in [16] and subsequently [12] and [15] in which they are called shuffle neighbourhoods. The authors commented that although these larger neighbourhoods were very time consuming and computationally intensive to use, the solutions they produced were significantly better and almost impossible to improve by hand. A very similar neighbourhood is also used by Valouxis and Housos [45] and later, Bellanti et al. [9] also describe a neighbourhood search which partially uses similar moves.

During development and testing, it was noticed that an expert human planner will often make improvements to a roster using similar moves. This may explain why an expert has such difficulty in improving a roster produced using this search neighbourhood.

\subsection{Comparing the Single Shift and Block Neighbourhood}

To examine how effective these neighbourhoods are and how quickly they can be searched using today's desktop personal computer, some experiments were conducted using a simple first improvement hill climber on the benchmark instances introduced in section 2. (Note that it is actually a hill descender as the penalty is being minimised but it is referred to as a hill climber as this is, generally, a more familiar term). The search uses neighbourhoods defined by swaps of up to a maximum length block of days. The pseudocode is given in Figure 5. This pseudocode is simply to provide an outline of the process. The actual implementation contains many more lines of code that increase efficiency and avoid redundancy (e.g. not visiting a solution already examined, etc).

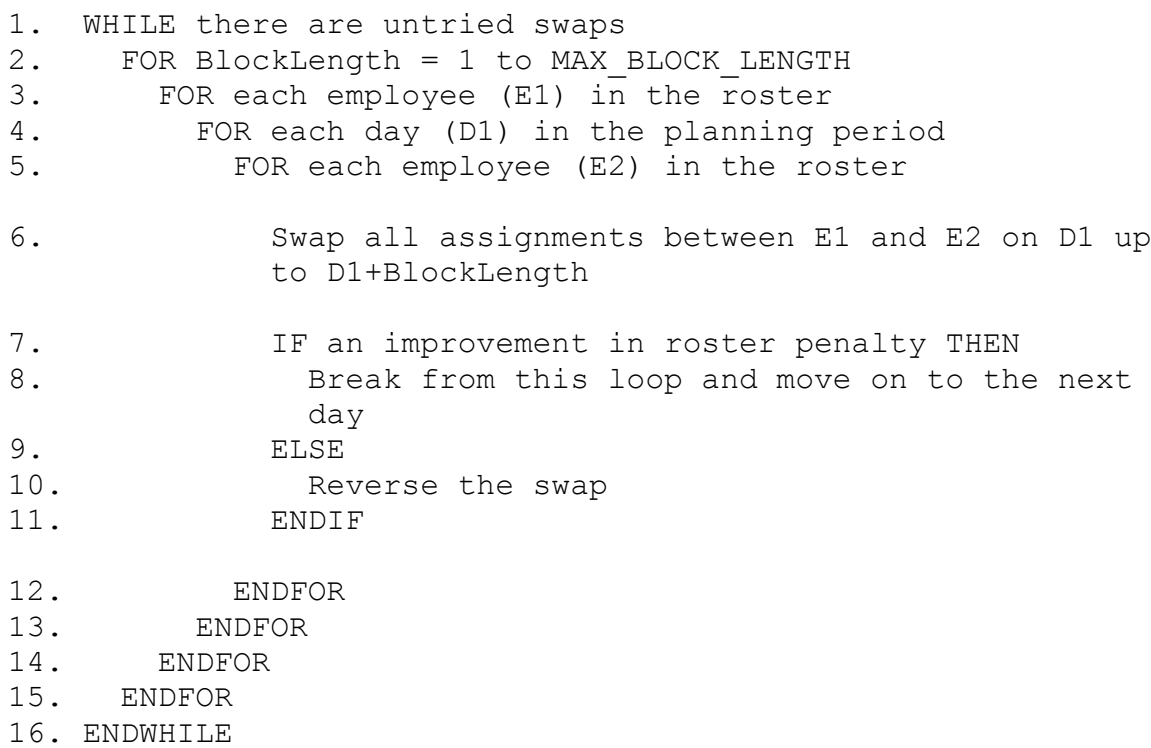

Figure 5 Pseudocode for the hill climber

The initial roster is created using a randomized greedy assignment method. It operates as follows: for each shift which needs to be covered, assign it to the nurse who incurs the least gain in penalty for their individual schedule (or who receives the greatest decrease in penalty) on receiving this shift.

In order to provide different starting solutions and allow the search to also be used with random restarts, the set of shifts to be assigned is randomly shuffled. The quality of the initial rosters created using this greedy algorithm is usually very poor. 
As there are few hard constraints, it is not difficult to construct a feasible roster. Also, the hard constraints are all related to coverage and it is possible to pre-calculate whether a feasible solution can be built. If a feasible solution does not exist, the user is notified that the cover requirements need to be reduced or extra staff need to be added. This is important as the hill climber operates over the feasible solution space.

Table 2 presents the results of the hill climber (outlined in Figure 5) when the maximum block length parameter (MBL) is set from one to ten. Note that when $\mathrm{MBL}=1$, it is effectively the single shift neighbourhood (assuming no more than one assignment per day).

Each experiment is repeated five times using different initial rosters (the same initial rosters are used for each MBL setting). The best, average and worst solutions, the average number of solutions examined per repeat and the average computation time per repeat are recorded for each instance. Table 2 contains the averages of these over all instances (for quick reference) and the results for each instance can be found in Table 3. The experiments were performed using a desktop PC with an Intel P4 $2.4 \mathrm{GHz}$ processor.

\begin{tabular}{rrrrrrr}
\hline Repeats & MBL & \multicolumn{1}{c}{ Best } & \multicolumn{1}{c}{ Average } & \multicolumn{1}{c}{ Worst } & $\begin{array}{c}\text { Average No. solutions } \\
\text { examined per repeat }\end{array}$ & $\begin{array}{r}\text { Average computation } \\
\text { time per repeat (secs) }\end{array}$ \\
\hline 5 & 1 & 2064 & 2820 & 3576 & 49200 & 3.8 \\
5 & 2 & 1565 & 2245 & 2614 & 86095 & 7.5 \\
5 & 3 & 1452 & 1840 & 2224 & 100472 & 9.2 \\
5 & 4 & 1413 & 1634 & 1893 & 115241 & 11.0 \\
5 & 5 & 1253 & 1573 & 2005 & 127452 & 12.4 \\
5 & 6 & 1191 & 1570 & 1891 & 138627 & 13.7 \\
5 & 7 & 1341 & 1526 & 1829 & 159760 & 16.3 \\
5 & 8 & 1160 & 1452 & 1652 & 171433 & 17.7 \\
5 & 9 & 1165 & 1462 & 1712 & 183773 & 19.2 \\
5 & 10 & 1165 & 1458 & 1721 & 185903 & 19.7 \\
25 & 1 & 1589 & 2613 & 3888 & 50513 & 4.0 \\
\hline
\end{tabular}

Table 2 Results of varying MAX_BLOCK_LENGTH (MBL)

\begin{tabular}{|c|c|c|c|c|c|c|c|c|c|c|c|c|c|}
\hline Instance & $\begin{array}{r}\text { Max } \\
\text { block } \\
\text { length }\end{array}$ & Best & Ave. & Worst & $\begin{array}{r}\text { Average } \\
\text { No. } \\
\text { solutions } \\
\text { examined }\end{array}$ & $\begin{array}{r}\text { Average } \\
\text { computation } \\
\text { time } \\
\text { (seconds) } \\
\end{array}$ & Instance & $\begin{array}{r}\text { Max } \\
\text { block } \\
\text { length } \\
\end{array}$ & Best & Ave. & Worst & $\begin{array}{r}\text { Average } \\
\text { No. } \\
\text { solutions } \\
\text { examined }\end{array}$ & $\begin{array}{r}\text { Average } \\
\text { omputation } \\
\text { time } \\
\text { (seconds) } \\
\end{array}$ \\
\hline ORTEC01 & 1 & 8927 & 14641 & 20192 & 55752 & 2.6 & BCV-5.4.1 & 1 & 487 & 725 & 933 & 1560 & 0.1 \\
\hline ORTEC01 & 2 & 5917 & 11046 & 13185 & 83462 & 4.1 & BCV-5.4.1 & 2 & 193 & 369 & 633 & 2389 & 0.1 \\
\hline ORTEC01 & 3 & 5120 & 7735 & 9771 & 109864 & 5.9 & BCV-5.4.1 & 3 & 48 & 196 & 487 & 2433 & 0.1 \\
\hline ORTEC01 & 4 & 5020 & 5706 & 6865 & 126689 & 7.1 & BCV-5.4.1 & 4 & 48 & 108 & 205 & 2670 & 0.1 \\
\hline ORTEC01 & 5 & 3355 & 5470 & 8045 & 144511 & 8.1 & BCV-5.4.1 & 5 & 48 & 106 & 195 & 2680 & 0.1 \\
\hline ORTEC01 & 6 & 2745 & 5303 & 6915 & 167063 & 9.7 & BCV-5.4.1 & 6 & 48 & 106 & 195 & 2831 & 0.1 \\
\hline ORTEC01 & 7 & 4257 & 4848 & 6470 & 173036 & 10.2 & BCV-5.4.1 & 7 & 48 & 106 & 195 & 3032 & 0.1 \\
\hline ORTEC01 & 8 & 2475 & 4022 & 4700 & 185363 & 11.1 & BCV-5.4.1 & 8 & 48 & 106 & 195 & 3268 & 0.1 \\
\hline ORTEC01 & 9 & 2525 & 4174 & 5295 & 202584 & 12.4 & BCV-5.4.1 & 9 & 48 & 106 & 195 & 3452 & 0.2 \\
\hline ORTEC01 & 10 & 2525 & 4131 & 5345 & 188767 & 11.6 & BCV-5.4.1 & 10 & 48 & 106 & 195 & 3587 & 0.2 \\
\hline BCV-1.8.1 & 1 & 328 & 491 & 698 & 9647 & 0.4 & BCV-6.13.1 & 1 & 1024 & 1324 & 1597 & 19883 & 1.0 \\
\hline BCV-1.8.1 & 2 & 291 & 448 & 650 & 14206 & 0.6 & BCV-6.13.1 & 2 & 1019 & 1223 & 1287 & 29736 & 1.6 \\
\hline BCV-1.8.1 & 3 & 288 & 332 & 470 & 20461 & 0.9 & BCV-6.13.1 & 3 & 994 & 1131 & 1286 & 34222 & 1.5 \\
\hline BCV-1.8.1 & 4 & 273 & 330 & 464 & 22138 & 1.1 & BCV-6.13.1 & 4 & 954 & 1057 & 1257 & 41324 & 2.4 \\
\hline BCV-1.8.1 & 5 & 287 & 338 & 470 & 22226 & 1.1 & BCV-6.13.1 & 5 & 954 & 1057 & 1257 & 46422 & 2. \\
\hline BCV-1.8.1 & 6 & 287 & 336 & 470 & 25884 & 1.3 & BCV-6.13.1 & 6 & 954 & 1057 & 1257 & 50864 & 3. \\
\hline
\end{tabular}




\begin{tabular}{|c|c|c|c|c|c|c|c|c|c|c|c|c|c|}
\hline BCV-1.8.1 & 7 & 287 & 337 & 471 & 30805 & 1.6 & BCV-6.13.1 & 7 & 954 & 1011 & 1101 & 60173 & 3.8 \\
\hline BCV-1.8.1 & 8 & 287 & 337 & 471 & 33242 & 1.8 & BCV-6.13.1 & 8 & 954 & 1011 & 1101 & 64769 & 4.2 \\
\hline BCV-1.8.1 & 9 & 287 & 329 & 471 & 35425 & 2.0 & BCV-6.13.1 & 9 & 954 & 1011 & 1101 & 69157 & 4.5 \\
\hline BCV-1.8.1 & 10 & 287 & 327 & 471 & 37639 & 2.1 & BCV-6.13.1 & 10 & 954 & 1011 & 1101 & 73355 & 4.9 \\
\hline BCV-2.46.1 & 1 & 1704 & 1715 & 1726 & 140592 & 12.4 & BCV-7.10.1 & 1 & 403 & 555 & 662 & 10259 & 0.4 \\
\hline BCV-2.46.1 & 2 & 1618 & 1674 & 1716 & 246792 & 24.1 & BCV-7.10.1 & 2 & 381 & 514 & 606 & 13863 & 0.6 \\
\hline BCV-2.46.1 & 3 & 1618 & 1663 & 1701 & 302478 & 31.4 & BCV-7.10.1 & 3 & 381 & 505 & 596 & 18048 & 0.9 \\
\hline BCV-2.46.1 & 4 & 1618 & 1663 & 1701 & 335523 & 35.8 & BCV-7.10.1 & 4 & 381 & 505 & 596 & 19851 & 1.1 \\
\hline BCV-2.46.1 & 5 & 1618 & 1663 & 1701 & 361902 & 39.5 & BCV-7.10.1 & 5 & 381 & 505 & 596 & 21480 & 1.1 \\
\hline BCV-2.46.1 & 6 & 1618 & 1663 & 1701 & 387107 & 42.8 & BCV-7.10.1 & 6 & 381 & 505 & 596 & 23038 & 1.2 \\
\hline BCV-2.46.1 & 7 & 1618 & 1663 & 1701 & 411298 & 46.4 & BCV-7.10.1 & 7 & 381 & 505 & 596 & 24540 & 1.3 \\
\hline BCV-2.46.1 & 8 & 1618 & 1663 & 1701 & 434607 & 49.8 & BCV-7.10.1 & 8 & 381 & 505 & 596 & 25982 & 1.4 \\
\hline BCV-2.46.1 & 9 & 1618 & 1663 & 1701 & 456895 & 52.9 & BCV-7.10.1 & 9 & 381 & 505 & 596 & 27368 & 1.6 \\
\hline BCV-2.46.1 & 10 & 1618 & 1663 & 1701 & 478249 & 55.9 & BCV-7.10.1 & 10 & 381 & 505 & 596 & 28691 & 1.7 \\
\hline BCV-3.46.1 & 1 & 3883 & 3969 & 4094 & 200929 & 16.8 & BCV-8.13.1 & 1 & 236 & 268 & 334 & 17229 & 0.8 \\
\hline BCV-3.46.1 & 2 & 3607 & 3675 & 3801 & 392147 & 37.2 & BCV-8.13.1 & 2 & 148 & 236 & 333 & 22756 & 1.1 \\
\hline BCV-3.46.1 & 3 & 3464 & 3525 & 3605 & 420806 & 41.7 & BCV-8.13.1 & 3 & 148 & 198 & 236 & 28734 & 1.5 \\
\hline BCV-3.46.1 & 4 & 3474 & 3507 & 3554 & 483621 & 49.7 & BCV-8.13.1 & 4 & 148 & 198 & 235 & 34321 & 1.9 \\
\hline BCV-3.46.1 & 5 & 3443 & 3463 & 3493 & 535836 & 56.8 & BCV-8.13.1 & 5 & 148 & 198 & 235 & 37471 & 2.1 \\
\hline BCV-3.46.1 & 6 & 3432 & 3463 & 3486 & 581235 & 62.8 & BCV-8.13.1 & 6 & 148 & 198 & 235 & 40455 & 2.3 \\
\hline BCV-3.46.1 & 7 & 3430 & 3456 & 3470 & 737642 & 82.7 & BCV-8.13.1 & 7 & 148 & 198 & 235 & 43316 & 2.5 \\
\hline BCV-3.46.1 & 8 & 3408 & 3446 & 3470 & 800863 & 90.5 & BCV-8.13.1 & 8 & 148 & 198 & 235 & 48029 & 2.9 \\
\hline BCV-3.46.1 & 9 & 3409 & 3446 & 3470 & 857518 & 98.4 & BCV-8.13.1 & 9 & 148 & 198 & 235 & 50671 & 3.1 \\
\hline BCV-3.46.1 & 10 & 3409 & 3454 & 3507 & 852724 & 99.3 & BCV-8.13.1 & 10 & 148 & 198 & 235 & 53218 & 3.3 \\
\hline BCV-4.13.1 & 1 & 75 & 110 & 189 & 12284 & 0.6 & BCV-A.12.1 & 1 & 3570 & 4397 & 5335 & 23867 & 2.9 \\
\hline BCV-4.13.1 & 2 & 17 & 53 & 75 & 20875 & 1.0 & BCV-A.12.1 & 2 & 2463 & 3210 & 3858 & 34728 & 4.6 \\
\hline BCV-4.13.1 & 3 & 22 & 54 & 75 & 21729 & 1.1 & BCV-A.12.1 & 3 & 2433 & 3060 & 4015 & 45941 & 6.4 \\
\hline BCV-4.13.1 & 4 & 22 & 54 & 75 & 24019 & 1.3 & BCV-A.12.1 & 4 & 2190 & 3207 & 3980 & 62257 & 9.2 \\
\hline BCV-4.13.1 & 5 & 22 & 52 & 74 & 31412 & 1.8 & BCV-A.12.1 & 5 & 2275 & 2878 & 3980 & 70575 & 10.4 \\
\hline BCV-4.13.1 & 6 & 22 & 52 & 74 & 33390 & 2.0 & BCV-A.12.1 & 6 & 2275 & 3019 & 3980 & 74401 & 11.2 \\
\hline BCV-4.13.1 & 7 & 22 & 52 & 74 & 35330 & 2.1 & BCV-A.12.1 & 7 & 2265 & 3082 & 3980 & 78429 & 11.9 \\
\hline BCV-4.13.1 & 8 & 15 & 50 & 74 & 38808 & 2.4 & BCV-A.12.1 & 8 & 2265 & 3178 & 3980 & 79397 & 12.3 \\
\hline BCV-4.13.1 & 9 & 13 & 49 & 74 & 45282 & 2.8 & BCV-A.12.1 & 9 & 2265 & 3134 & 3980 & 89375 & 14.0 \\
\hline BCV-4.13.1 & 10 & 13 & 49 & 74 & 47293 & 3.0 & BCV-A.12.1 & 10 & 2265 & 3134 & 3980 & 95509 & 15.0 \\
\hline
\end{tabular}

Table 3 Results of varying MBL in the hill climber (5 repeats for each instance)

\begin{tabular}{lrrrrrr}
\hline & & & & & $\begin{array}{r}\text { Average No. } \\
\text { solutions }\end{array}$ & $\begin{array}{r}\text { Average } \\
\text { computation } \\
\text { examined }\end{array}$ \\
\hline time (seconds)
\end{tabular}

Table 4 Hill climber, 25 repeats with $\mathrm{MBL}=1$ 
The results in Table 3 and Table 2 show that the single shift neighbourhood (i.e. $\mathrm{MBL}=1$ ) is not as effective as when MBL $>1$. It is logical to question whether this is simply due to less solutions being examined when $M B L=1$. To provide a fairer comparison, the experiments were repeated for $\mathrm{MBL}=1$ but with 25 instead of 5 repeats (Table 4 and the bottom row of Table 2). This ensures that the searches with MBL $=1$ receive at least the same time (and in most cases more) as each experiment with MBL $>1$. Although giving a longer computation time did improve the best solution found, it was still worse on all instances than $\mathrm{MBL}=2$ and significantly worse than MBL $>5$.

As shown, increasing MBL increases the quality of the results but at the cost of extra computation time. However, when MBL $>8$, any increases in performance become less clear. Although MBL could range up to the number of days in the planning period, the results suggest that setting MBL $>8$ does not yield better results, especially in relation to the extra computation time required. In fact, when $M B L>8$ the results deteriorate slightly for some instances. This would have been a strange result if line 1 of the pseudocode was not present. However, what is happening is that a move is being made when the block length $=9$ that would obviously not have been made if $\mathrm{MBL}<9$ and hence in the next iteration of the loop at line 1 the current solution is slightly different. It can also be seen that the increase in computation time is approximately linear in relation to MBL.

On average, increasing MBL will yield better solutions. However, if the results for each instance are studied, the benefits of using larger blocks on some instances is less noticeable. For example, on instances BCV-2.46.1 and BCV-7.10.1, $\mathrm{MBL}=2$ is only slightly better than $\mathrm{MBL}=1$ and increasing MBL above 3 gives no further improvement. Similarly, increasing MBL above 5 for instance BCV-5.4.1 is not worthwhile. Although the reasons for this are not obvious, it is thought to be linked to which types of constraints and their priorities are used in the schedules. Although it may be possible to estimate the suitability of neighbourhoods for a particular instance based on its constraints, it would also be difficult, as potentially each nurse could request a different set of constraint types with different parameters for any one scheduling period.

The computation times for each instance range from $<1$ second to approximately 90 seconds. As would be expected, the longer computation times are required for the rosters with more employees, longer planning horizons and also those instances which utilise a larger set of the available soft constraint types for each employee (e.g instance BCV-A.12.1).

As can be seen, on the machine used, the search, on average, examines approximately 10,000 solutions per second. Examining the results for each instance reveals that the solutions examined per second ranges from approximately 28,000 for the instances with fewer soft constraints to around 6,000 for the instances which use all the available soft constraint types. Evaluating soft constraints is by far the most time consuming function in the search and so a large amount of effort was spent streamlining them to ensure that they were fast and efficient as well as accurate. It is possible to obtain large increases in search performance through writing faster code than any new heuristic or search mechanism may be able to achieve. This is not always appreciated and the challenge and importance of writing fast and efficient evaluation functions is often underestimated. Also, metrics such as the performance 
per number of solutions evaluated are often analysed less, if at all. In the results, the number of rosters examined as well as computation times are provided. In the authors' opinion this is a more revealing, reliable and future-proof measure of a search method's performance.

\section{The Variable Depth Search}

As the results in the previous section show, using today's average desktop PC, a local search employing the block neighbourhood can be completed on the larger instances in less than 90 seconds. These solutions are very difficult to improve by hand. However, due to the complexity of the problems, they are still very often local optima (albeit high quality ones). Therefore, end users may wish to use idle computer time (e.g. during a lunch break or over night) to try and find even higher quality rosters. Perhaps the simplest way to provide this option is by restarting the hill climber as many times as possible in the allotted time with different initial rosters, in the form of a basic iterated local search [33]. This is something that was tested and the results are provided in section 5. However, the main focus of this chapter is a variable depth search which will now be introduced.

The first step of the algorithm is to create an initial roster. This is done using the greedy assignment method introduced in section 3.3. As mentioned, these initial rosters can be constructed very quickly (in less than a second) but are generally of poor quality. It was found, however, that the quality of the initial roster had relatively little impact on the final roster. Once the initial roster is created, it is possible to proceed with the variable depth search which, like the hill climber, also operates over the feasible solution space. Figure 6 provides an outline of the algorithm.

The search is similar to a method used when attempting to manually improve rosters. When improving rosters by hand it was observed that first we would try and improve one nurse's individual schedule (that is lower the penalty for that nurse's schedule). Improving this nurse's schedule would usually be at the expense of another nurse so we then try and improve their schedule. If the second nurse's schedule is improved it may be at the expense of a third nurse's schedule so we then move on to the third nurse and so on until (hopefully) we have an overall roster penalty that is lower than the original penalty. If not, we would reverse all the changes we have just made and try a different path. This is the basic idea behind the algorithm. 


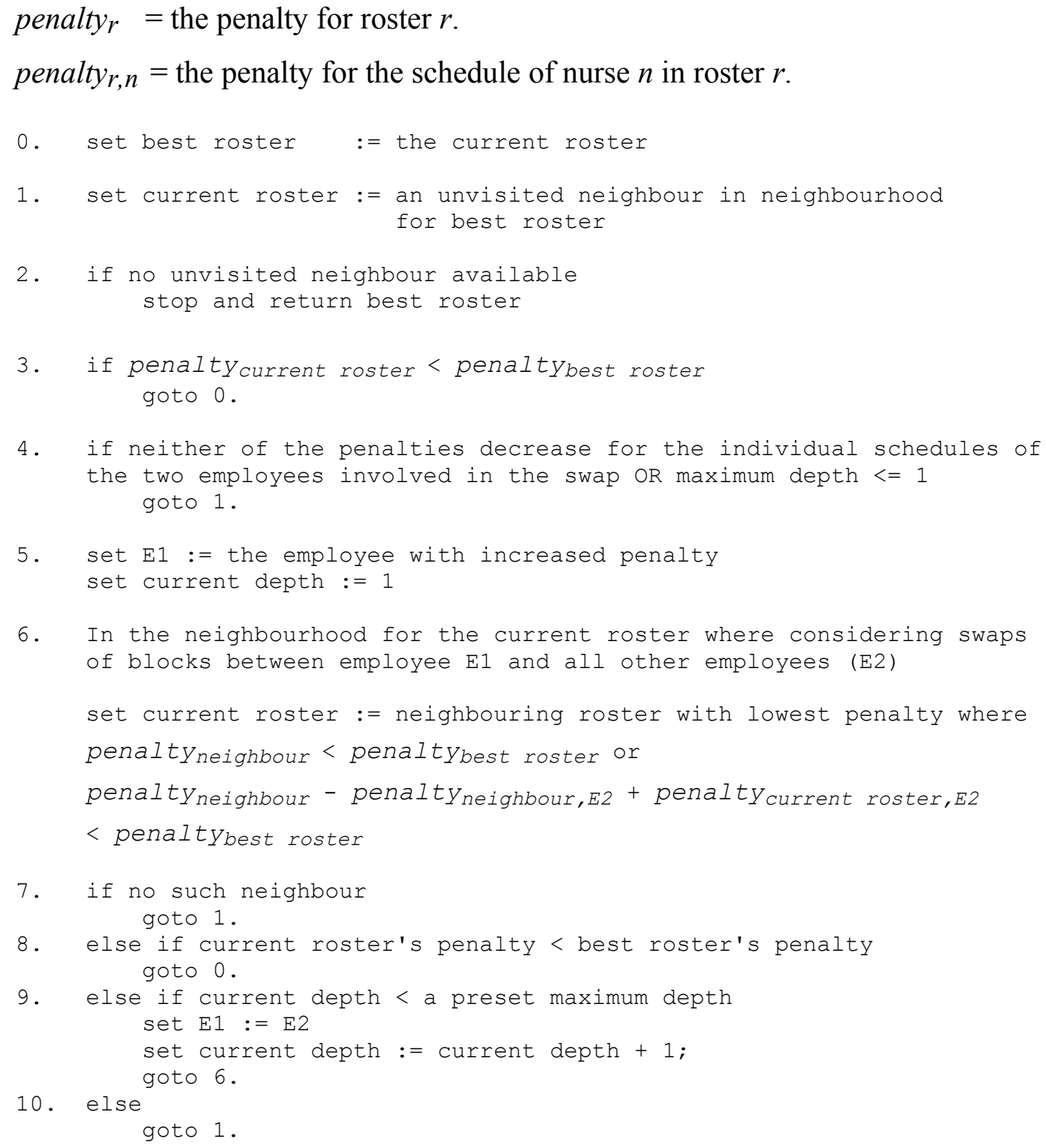

Figure 6 Variable depth search outline

The neighbourhoods referred to in Figure 6 are identified by swaps of blocks up to a maximum block length (MBL). The neighbourhood at step 1 is defined by all possible swaps of blocks, on all days of the planning period, between all nurses. At step 6, the swaps are just between two nurses on all days of the planning period. It was found to be generally more efficient to set MBL at step 1 lower than at step 6 (e.g at step 1, use 2 or 3 and at step 6 , use 5 or 6 ).

At various points in the algorithm (e.g. steps 4 and 6), it is necessary to analyse the change in penalty for a nurse's individual schedule after a swap has been performed. After any swap, at least one but no more than two of the nurse's individual schedules will have been altered. However, the penalties for other nurses' schedules may also have changed even though their schedule has not been modified. This occurs in instances which use the so called 'vertical' constraints of tutorship and ensuring that certain nurses work separately. Therefore, when analysing the change in penalty for any individual nurse's schedule that has just been altered, what we actually use is the 
net change in penalties for this nurse and all other nurses that are directly linked to this nurse by 'vertical' constraints.

\subsection{Heuristics}

It was stated that the search operates over the feasible solution space. However, it was discovered that it was beneficial to treat the hard constraint that a nurse must have the skills required to perform a shift as a soft constraint. This can be achieved by assigning a sufficiently high weight to the hard constraint violation (e.g. giving it the same value as the penalty of the initial roster) thus ensuring that a solution with this constraint violated will not be returned. Using it as a soft constraint though, allows greater exploration of the search space. This happens because rosters with this hard constraint violated are sometimes used as intermediate solutions in a chain of moves and a better local optimum may be reached via them.

Step 6 is perhaps the most important step in the algorithm. Step 6 specifies which moves to examine as potential candidates to be added to the current chain of moves and also defines the rule for deciding which one (if any) to select. As outlined in Figure 6, a swap is only selected as a potential move to add to the current chain if ignoring the change in N2's penalty, the neighbour's penalty is less than the best roster's penalty. This rule is similar to, and inspired by, the 'Gain Criterion' of the Lin-Kernighan algorithm for the travelling salesman problem [32]. In section 5, the results of a number of experiments are presented in which this rule is removed to investigate its benefit.

The number of moves to examine in order to select candidates for continuing the chain can have a significant effect on the performance of the algorithm. If too many moves are tested, then the algorithm's run time will increase. If too few are selected, then there is a smaller chance of a successful one being found and the algorithm will become less effective. In Figure 6, all swaps up to a maximum block length, on all days of the planning period, between one nurse and all the others are tested. Reducing the run time by limiting the number of nurses to test swaps between and reducing the number of days adjacent to the swap at step 1 over which to test swaps was evaluated. As expected, the run time was improved but at the cost of roster quality. To try and increase efficiency, two heuristics for selecting candidate moves were developed and tested instead.

In the first heuristic (violation flag heuristic), all days which need changing either through the removal, addition or changing of shift assignments, in order to remove a soft constraint violation are flagged during penalty recalculations. Only the swaps which involve at least one of these days are then tested. This heuristic is also applied at step 1. Only focusing on parts of a solution that have violations and need repairing is a common heuristic. For example, Nonobe and Ibaraki [40] use a similar heuristic in a tabu search approach tested on a nurse rostering problem formulated as a constraint satisfaction problem.

In the second heuristic (worsened days heuristic), an array of penalties due to soft constraint violations for each day is maintained for each nurse's schedule during penalty calculations. Using these arrays, the moves in step 6 are then restricted to only those blocks that contain days which were made worse (i.e. penalty increased) after 
the last move. This is a more restrictive heuristic as days which contain violations will be ignored if they were not affected by the last swap in the chain.

\subsection{Pre-defined Run Time}

The running time for the algorithm depends on the size of the neighbourhoods at steps 1 and 6, the maximum depth used at step 9 and the structure of the instance being addressed. The size of the neighbourhoods at steps 1 and 6 depends upon the number of nurses, the number of days in the planning period and the maximum block length. The effects of the third factor (the instance structure) on the running time cannot be as easily predicted as factors such as the number of nurses and days. For some instances, it is possible that the structure (determined more by the soft constraints and their weights) is such that there is very often a valid neighbour found at step 6 with which to replace the current roster but which is not better than the best roster. This can mean that the search sometimes reaches great depths which obviously affects the running time.

To reduce this effect, a maximum depth which is set beforehand is used at step 9 . Initially the depth was set using a trial and error method of running the algorithm for a short time and observing its progress on the particular instance. Then altering the maximum depth value until a suitable setting is found (that is estimated) will restrict the algorithm to a satisfactory running time. This is obviously not a suitable approach for practical use. Therefore, an additional mechanism was added which takes the preferred running time as a parameter and attempts to use that time efficiently.

This mechanism works as follows: for the algorithm to finish, every neighbour in the neighbourhood at step 1 needs to be examined and potentially used as the first solution in a chain of moves. It is possible to calculate the size of the neighbourhood at step one using the number of nurses, the maximum block length and the number of days in the planning horizon. Given a preferred running time and the number of solutions to evaluate at step one (updated each time a new best solution is found), it is possible to calculate an average time to spend using each neighbour at step one as the first solution in a chain. Then at step 9, instead of testing whether a maximum depth will be exceeded in continuing the chain, we test whether the average time per chain will be exceeded if it continues.

In the results section where this heuristic is not used but a maximum running time is set, the search immediately terminates and returns the best solution when the time limit is reached. If the algorithm naturally terminates and the preferred running time has not been exceeded, then at step 2, instead of returning the best roster, a new initial roster is created and the algorithm restarts at step 0.

Figure 7 shows an example of an improving chain of moves. The change in the roster consists of seven moves which, when performed simultaneously, provide an overall reduction in the roster's penalty. It can be seen that the second nurse of a swap is always the first nurse in the next swap. Note that Figure 7 is just used to illustrate the idea of a chain of moves. For the roster shown (which is far from optimal), there are many other chains which would also improve the roster. 


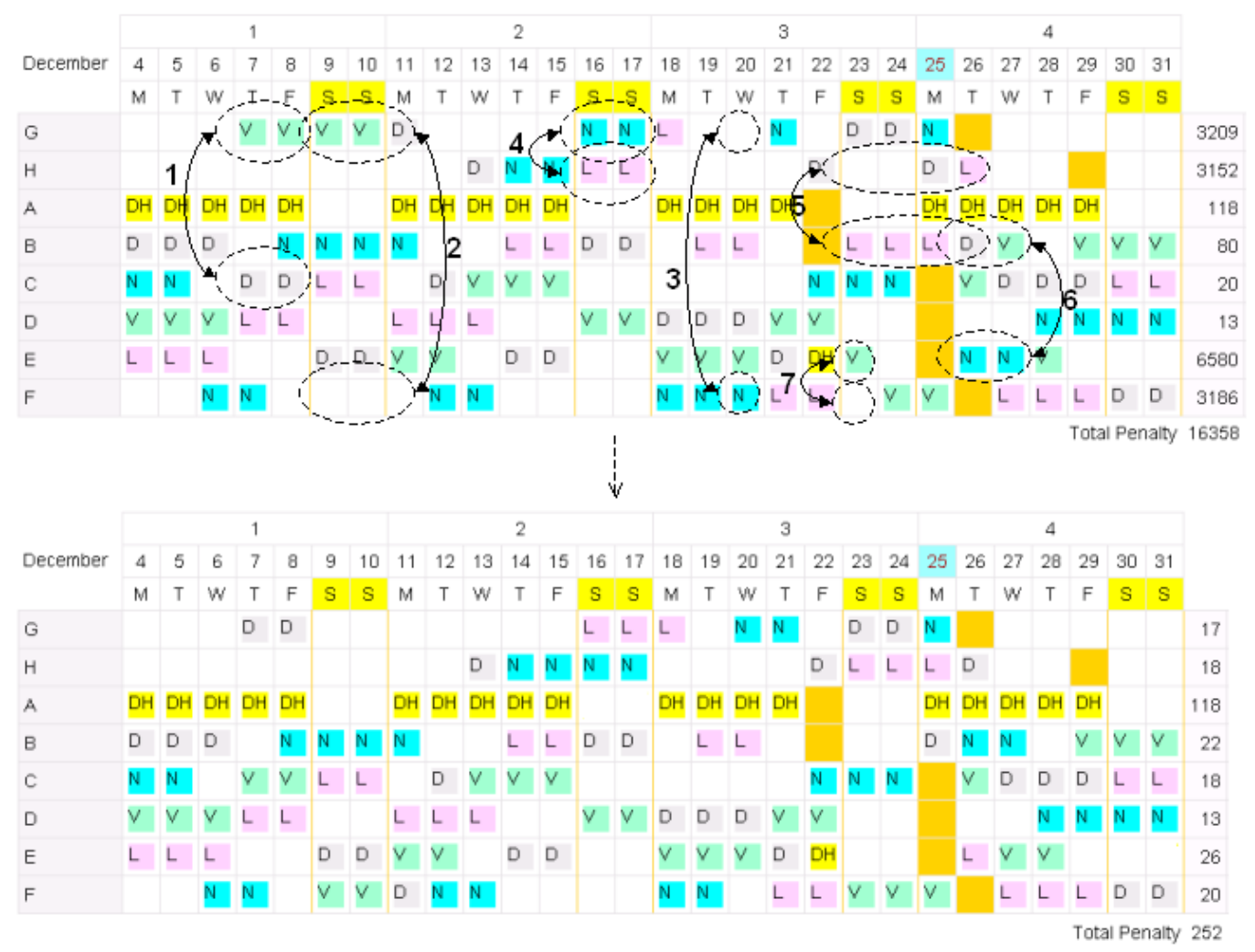

Figure 7 Example chains of swaps

From observing the logs after searches, the lengths of improving chains of moves varies greatly. Note that poor rosters are easily improved by single moves. When the local optima start to be found less frequently, (and the penalty is approaching better values) even for the smaller instances, improving chains of moves with lengths over one hundred are not uncommon.

\subsection{Efficient Implementation}

At step 6, there is a possibility that a neighbouring solution will be selected that has been visited previously and cycling could occur. Two different methods were tested to remove this risk. In the first method, a history of solutions visited along the current path (i.e. since the last use of step 1) is maintained and used to ensure that they are not revisited. This is fast and prevents cycling but does not guarantee that solutions are not revisited at other points in the algorithm, for example visiting a solution at step 1 that has already been used at step 6 . The second method maintains a hash table of all solutions visited during the run of the algorithm and so also prevents all solutions being revisited. Testing showed that the first, simpler method, produced better results. This appears to be because the probability of visiting a duplicate solution at the points which the first method does not prevent is small and much lower than the probability of cycling at step 6 . Therefore, using the faster method which prevents the majority of cycling and revisiting duplicate solutions was more efficient than the slower approach which guaranteed no cycling or duplicate paths.

As discussed earlier, increases in performance can be achieved as effectively through making the algorithm faster and more efficient as by using better heuristics. We have already mentioned the importance of avoiding cycling. There are also some other 
efficiency measures which are worth highlighting. Firstly, when a nurse's schedule has been altered it is only necessary to re-evaluate their schedule and any other nurses' schedules which may be linked by vertical constraints to recalculate the new roster's penalty. Secondly, by far the most time consuming operation is calculating penalties (i.e. soft constraint evaluations). If there is a likelihood that a solution will be returned to, then the algorithm caches penalties to avoid having to recalculate them. Finally, some soft constraint calculations can be speeded up by using data structures that are modified as assignments are made. A simple example is to update the total number of hours worked when a shift is (un)assigned rather than to add all the hours up when calculating the penalty. Some of the more complicated constraints benefit from a similar approach.

\section{Results}

The algorithm was tested on the ten publicly available data sets introduced in section 2.

\subsection{Comparing Heuristics}

In the experiments, the following parameters were used: Maximum depth $=1000$, maximum block length at step $1=2$, maximum block length at step $6=5$. As described, if the algorithm finishes before the maximum running time is reached, a new initial solution is constructed and the search restarts.

Table 5, Table 6, Table 7 and Table 8 contain the results of the variable depth search when different heuristics are used. Predefined maximum run times of 1 minute, 2 minutes and 5 minutes were tested with each instance and repeated five times using different random seeds (but which were the same for each corresponding trial). The best, worst and average of these runs were recorded. The penalties in Table 5 are the averages over all instances. The results for each instance given in Table 6, Table 7 and Table 8.

The heuristics are summarised below:

No heuristics Step 4 is removed and step 5 is applied twice but with E1 set as the other nurse each time. At step 6 the only rule is to select the best neighbour.

PG Partial gain heuristic (only add a swap to the end of the current chain if it satisfies the partial improvement criterion).

VF Violation flag heuristic (only test swaps which include at least one day which is flagged as having a violation). This is used at steps 1 and 6.

WD Only swap worsened days heuristic (only test swaps which include at least one day which has a violation and the violation's penalty was increased or the violation occurred after the last swap). This is used at step 6.

TR Time restriction heuristic (ensure that a pre-calculated average amount of time is spent on every chain).

ILS Hill climber with restarts. Maximum depth is set to 1 and maximum block size at step 1 is increased to 5 .

\begin{tabular}{l|ccc|ccc|ccc}
\hline & \multicolumn{3}{|c|}{ 1 Minute } & \multicolumn{3}{c|}{ 2 Minutes } & \multicolumn{3}{c}{ 5 Minutes } \\
\hline Heuristics & Best & Avg. & Worst & Best & Avg. & Worst & Best & Avg. & Worst \\
\hline No heuristics & 1390 & 1561 & 1879 & 1196 & 1454 & 1825 & 1154 & 1394 & 1766
\end{tabular}




\begin{tabular}{l|rrr|rrr|rrr} 
PG & 917 & 1156 & 1461 & 888 & 1078 & 1271 & 872 & 1034 & 1225 \\
VF & 1222 & 1629 & 2050 & 1113 & 1329 & 1611 & 973 & 1111 & 1232 \\
WD & 1046 & 1241 & 1515 & 1008 & 1131 & 1251 & 972 & 1087 & 1234 \\
PG + VF & 1003 & 1228 & 1680 & 894 & 1163 & 1619 & 872 & 1016 & 1303 \\
PG + WD & 900 & 1016 & 1205 & 895 & 973 & 1083 & 877 & 946 & 1031 \\
TR & 1153 & 1454 & 1829 & 1175 & 1450 & 1654 & 1008 & 1232 & 1386 \\
TR + PG + VF & 1014 & 1122 & 1277 & 888 & 994 & 1141 & 874 & 978 & 1141 \\
TR + PG + WD & 899 & 1068 & 1260 & 862 & 935 & 1027 & 858 & 889 & 938 \\
ILS & 1164 & 1266 & 1405 & 1048 & 1174 & 1290 & 1012 & 1067 & 1161 \\
\hline
\end{tabular}

Table 5 Comparison of heuristic combinations

\begin{tabular}{|c|c|c|c|c|c|c|c|c|c|c|c|}
\hline & & & & $=1000$ &. & & & & & & \\
\hline & & $\begin{array}{c}\text { No } \\
\text { heuristics }\end{array}$ & PG & VF & WD & $\begin{array}{c}\mathrm{PG}+ \\
\mathrm{VF}\end{array}$ & $\begin{array}{l}\mathrm{PG}+ \\
\mathrm{WD}\end{array}$ & TR & $\begin{array}{c}\mathrm{TR}+ \\
\mathrm{PG}+ \\
\mathrm{VF}\end{array}$ & $\begin{array}{c}\text { TR + } \\
\text { PG + } \\
\text { WD }\end{array}$ & ILS \\
\hline ORTEC01 & Average & 5768 & 2358 & 6560 & 3234 & 3347 & 1103 & 4605 & 2242 & 1910 & 3573 \\
\hline & Best & 4826 & 506 & 3245 & 1655 & 1421 & 425 & 2746 & 1430 & 555 & 2825 \\
\hline BCV-1.8.1 & Average & 263 & 263 & 267 & 265 & 263 & 259 & 278 & 257 & 261 & 266 \\
\hline & Best & 255 & 253 & 259 & 257 & 254 & 254 & 271 & 254 & 255 & 261 \\
\hline BCV-2.46.1 & Average & 1679 & 1667 & 1643 & 1662 & 1659 & 1683 & 1657 & 1605 & 1598 & 1641 \\
\hline & Best & 1653 & 1616 & 1595 & 1633 & 1616 & 1646 & 1618 & 1593 & 1574 & 1618 \\
\hline BCV-3.46.1 & Average & 3570 & 3478 & 3546 & 3515 & 3501 & 3481 & 3588 & 3477 & 3411 & 3464 \\
\hline & Best & 3483 & 3411 & 3456 & 3505 & 3460 & 3422 & 3531 & 3442 & 3380 & 3443 \\
\hline BCV-4.13.1 & Average & 11 & 13 & 10 & 11 & 11 & 10 & 22 & 11 & 11 & 12 \\
\hline & Best & 10 & 10 & 10 & 10 & 10 & 10 & 11 & 10 & 10 & 11 \\
\hline BCV-5.4.1 & Average & 48 & 48 & 48 & 48 & 48 & 48 & 48 & 48 & 48 & 48 \\
\hline & Best & 48 & 48 & 48 & 48 & 48 & 48 & 48 & 48 & 48 & 48 \\
\hline BCV-6.13.1 & Average & 941 & 873 & 793 & 770 & 814 & 769 & 844 & 768 & 770 & 908 \\
\hline & Best & 827 & 768 & 768 & 768 & 768 & 768 & 769 & 768 & 768 & 822 \\
\hline BCV-7.10.1 & Average & 419 & 455 & 385 & 384 & 383 & 384 & 392 & 384 & 384 & 381 \\
\hline & Best & 381 & 381 & 381 & 381 & 381 & 381 & 381 & 381 & 381 & 381 \\
\hline BCV-8.13.1 & Average & 180 & 164 & 148 & 148 & 148 & 148 & 196 & 148 & 148 & 148 \\
\hline & Best & 148 & 148 & 148 & 148 & 148 & 148 & 148 & 148 & 148 & 148 \\
\hline BCV-A.12.1 & Average & 2731 & 2240 & 2890 & 2370 & 2103 & 2271| & 2912 & 2284 & 2135 & 2215 \\
\hline & Best & 2265 & 2033 & 2309 & 2050 & 1919 & 1900 & 2009 & 2068 & 1870 & 2085 \\
\hline
\end{tabular}

Table 6 Variable depth search heuristics with max. run time 1 minute

\begin{tabular}{|c|c|c|c|c|c|c|c|c|c|c|c|}
\hline & & $\begin{array}{c}\text { No } \\
\text { heuristics }\end{array}$ & PG & $\mathrm{VF}$ & WD & $\begin{array}{c}\mathrm{PG}+ \\
\mathrm{VF} \\
\end{array}$ & $\begin{array}{l}\mathrm{PG}+ \\
\mathrm{WD}\end{array}$ & TR & $\begin{array}{c}\mathrm{TR}+ \\
\mathrm{PG}+ \\
\mathrm{VF} \\
\end{array}$ & $\begin{array}{l}\mathrm{TR}+ \\
\mathrm{PG}+ \\
\mathrm{WD}\end{array}$ & ILS \\
\hline \multirow[t]{2}{*}{ ORTEC01 } & Average & 5023 & 1931 & 4246 & 2529 & 2893 & 860 & 4990 & 1262 & 868 & 2713 \\
\hline & Best & 3110 & 485 & 2450 & 1605 & 520 & 425 & 3099 & 470 & 480 & 1691 \\
\hline \multirow[t]{2}{*}{ BCV-1.8.1 } & Average & 263 & 261 & 265 & 262 & 260 & 255 & 269 & 257 & 257 & 265 \\
\hline & Best & 255 & 252 & 257 & 257 & 254 & 253 & 263 & 253 & 253 & 261 \\
\hline \multirow[t]{2}{*}{ BCV-2.46.1 } & Average & 1679 & 1664 & 1642 & 1652 & 1649 & 1680 & 1627 & 1597 & 1600 & 1620 \\
\hline & Best & 1653 & 1616 & 1595 & 1633 & 1596 & 1646 & 1616 & 1572 & 1574 & 1615 \\
\hline \multirow[t]{2}{*}{ BCV-3.46.1 } & Average & 3486 & 3433 & 3477 & 3433 & 3477 & 3457 & 3523 & 3410 & 3380 & 3446 \\
\hline & Best & 3449 & 3392 & 3419 & 3414 & 3399 & 3372 & 3479 & 3380 & 3338 & 3413 \\
\hline \multirow[t]{2}{*}{ BCV-4.13.1 } & Average & 11 & 11 & 10 & 11 & 10 & 10 & 11 & 11 & 10 & 12 \\
\hline & Best & 10 & 10 & 10 & 10 & 10 & 10 & 10 & 10 & 10 & 11 \\
\hline
\end{tabular}




\begin{tabular}{ll|r|r|r|r|r|r|r|r|r|r} 
BCV-5.4.1 Average & 48 & 48 & 48 & 48 & 48 & 48 & 48 & 48 & 48 & 48 \\
& Best & 48 & 48 & 48 & 48 & 48 & 48 & 48 & 48 & 48 & 48 \\
BCV-6.13.1 Average & 904 & 842 & 780 & 769 & 768 & 769 & 836 & 768 & 768 & 889 \\
& Best & 827 & 768 & 768 & 768 & 768 & 768 & 774 & 768 & 768 & 822 \\
BCV-7.10.1 & Average & 419 & 437 & 384 & 384 & 382 & 384 & 403 & 384 & 384 & 381 \\
Best & 381 & 381 & 381 & 381 & 381 & 381 & 381 & 381 & 381 & 381 \\
BCV-8.13.1 & Average & 164 & 148 & 148 & 148 & 148 & 148 & 148 & 148 & 148 & 148 \\
Best & 148 & 148 & 148 & 148 & 148 & 148 & 148 & 148 & 148 & 148 \\
BCV-A.12.1 & Average & 2540 & 2007 & 2293 & 2071 & 1992 & 2115 & 2647 & 2051 & 1882 & 2213 \\
Best & 2075 & 1775 & 2050 & 1819 & 1820 & 1900 & 1928 & 1845 & 1620 & 2085 \\
\hline
\end{tabular}

Table 7 Variable depth search heuristics with max. run time 2 minutes

\begin{tabular}{|c|c|c|c|c|c|c|c|c|c|c|c|}
\hline \multicolumn{12}{|c|}{$\begin{array}{l}\text { Time restriction }=5 \text { minutes. Max depth }=1000 . \text { Max. block length at step } 6=5 . \text { Max. block length at } \\
\text { step } 1=2 .\end{array}$} \\
\hline & & $\begin{array}{c}\text { No } \\
\text { heuristics }\end{array}$ & PG & VF & WD & $\begin{array}{c}\mathrm{PG}+ \\
\mathrm{VF} \\
\end{array}$ & $\begin{array}{l}\mathrm{PG}+ \\
\mathrm{WD}\end{array}$ & TR & $\begin{array}{c}\mathrm{TR}+ \\
\mathrm{PG}+ \\
\mathrm{VF}\end{array}$ & $\begin{array}{c}\mathrm{TR}+ \\
\mathrm{PG}+ \\
\mathrm{WD} \\
\end{array}$ & ILS \\
\hline \multirow[t]{2}{*}{ ORTEC01 } & Average & 4763 & 1785 & 2421 & 2234 & \begin{tabular}{|l|}
1570 \\
\end{tabular} & 857 & 3150 & 1147 & 448 & 1901 \\
\hline & Best & 3000 & 460 & 1390 & 1410 & 435 & 425 & 1420 & 360 & 420 & 1590 \\
\hline \multirow[t]{2}{*}{ BCV-1.8.1 } & Average & 259 & 258 & 262 & 255 & 255 & 254 & 264 & 257 & 254 & 263 \\
\hline & Best & 254 & 252 & 256 & 253 & 253 & 253 & 255 & 253 & 253 & 257 \\
\hline \multirow[t]{2}{*}{ BCV-2.46.1 } & Average & 1664 & 1654 & 1621 & 1635 & 1647 & 1669 & 1622 & 1596 & 1586 & 1607 \\
\hline & Best & 1633 & 1614 & 1595 & 1594 & 1595 & 1613 & 1595 & 1572 & 1572 & 1595 \\
\hline \multirow[t]{2}{*}{ BCV-3.46.1 } & Average & 3468 & 3404 & 3465 & 3392 & 3448 & 3423 & 3443 & 3392 & 3378 & 3427 \\
\hline & Best & 3427 & 3374 & 3411 & 3382 & 3399 & 3372 & 3399 & 3347 & 3355 & 3413 \\
\hline \multirow[t]{2}{*}{ BCV-4.13.1 } & Average & 11 & 11 & 10 & 10 & 10 & 10 & 12 & 10 & 10 & 11 \\
\hline & Best & 10 & 10 & 10 & 10 & 10 & 10 & 10 & 10 & 10 & 10 \\
\hline \multirow[t]{2}{*}{ BCV-5.4.1 } & Average & 48 & 48 & 48 & 48 & 48 & 48 & 48 & 48 & 48 & 48 \\
\hline & Best & 48 & 48 & 48 & 48 & 48 & 48 & 48 & 48 & 48 & 48 \\
\hline \multirow[t]{2}{*}{ BCV-6.13.1 } & Average & 857 & 818 & 768 & 769 & 768 & 768 & 814 & 768 & 768 & 853 \\
\hline & Best & 768 & 768 & 768 & 768 & 768 & 768 & 770 & 768 & 768 & 777 \\
\hline \multirow[t]{2}{*}{ BCV-7.10.1 } & Average & 401 & 401 & 382 & 383 & 382 & 383 & 384 & 383 & 383 & 381 \\
\hline & Best & 381 & 381 & 381 & 381 & 381 & 381 & 381 & 381 & 381 & 381 \\
\hline \multirow[t]{2}{*}{ BCV-8.13.1 } & Average & 148 & 148 & 148 & 148 & 148 & 148 & 148 & 148 & 148 & 148 \\
\hline & Best & 148 & 148 & 148 & 148 & 148 & 148 & 148 & 148 & 148 & 148 \\
\hline \multirow[t]{2}{*}{ BCV-A.12.1 } & Average & 2320 & 1814 & 1985 & 2000 & $\mid 1881$ & 1900 & 2437 & 2028 & 1867 & 2034 \\
\hline & Best & 1869 & 1664 & 1720 & 1729 & 1679 & 1750 & 2053 & 1849 & 1620 & 1900 \\
\hline
\end{tabular}

Table 8 Variable depth search heuristics with max. run time 5 minutes

The results indicate that the best combination of heuristics is TR+PG+WD. The better results all use PG. TR is improved if combined with other heuristics and similarly VF and WD also perform better when not used on their own. As would be expected, VF and WD perform similarly (WD is a more restrictive form of VF). ILS performs similarly to TR, VF and WD when they are used on their own. All other combinations outperform ILS.

Examining the results for each instance is also interesting. VF is very effective on instance BCV-4.13.1, even when used on its own and WD+PG works well on instance ORTEC01. However, we were unable to draw any definite conclusions on why this should be the case after examining the characteristics of these instances more closely. Due to its simplicity ILS is able to examine more solutions in the allocated time but its lack of heuristics makes it less effective. 


\subsection{Comparisons with Other Methods}

Brucker et al. [11] developed a heuristic constructive approach and tested it on the benchmark instances. As it is a constructive method it is not possible to provide a comparison to the variable depth search by using the number of solutions examined metric. However, their experiments were performed on the same machine and a comparison can be provided by using computation times. The results in Table 9 are Brucker et al's best results from all experiments. The total computation time in obtaining these solutions for each instance was then set as the maximum run time for the variable depth search with the heuristic combination TR+PG+VF.

Burke et al's result for ORTEC01 using the hybrid variable neighbourhood search [13] had a computation time of 12 hours. The result for the variable depth search on this instance is the best of the five, five minute tests using the heuristic combination $\mathrm{TR}+\mathrm{PG}+\mathrm{VF}$.

As can be seen, the variable depth search outperforms the constructive method, over the same computation times, on all instances except one, on which they are equal. It also beats the hybrid method of Burke et al. on instance ORTEC01.

\begin{tabular}{l|r|r|r|r}
\hline & \multicolumn{1}{|c|}{ Time } & Brucker et al. [11] & Burke et al. [13] & Variable Depth Search \\
\hline ORTEC01 & $12 \mathrm{hrs}$ & 541 & 360 \\
BCV-1.8.1 & $136 \mathrm{sec}$ & 323 & - & 253 \\
BCV-2.46.1 & $3424 \mathrm{sec}$ & 1594 & - & 1572 \\
BCV-3.46.1 & $2888 \mathrm{sec}$ & 3601 & - & 3324 \\
BCV-4.13.1 & $208 \mathrm{sec}$ & 18 & - & 10 \\
BCV-5.4.1 & $16 \mathrm{sec}$ & 200 & - & 48 \\
BCV-6.13.1 & $304 \mathrm{sec}$ & 890 & - & 768 \\
BCV-7.10.1 & $216 \mathrm{sec}$ & 396 & - & 381 \\
BCV-8.13.1 & $224 \mathrm{sec}$ & 148 & - & 148 \\
BCV-A.12.1 & $944 \mathrm{sec}$ & 3335 & - & 1843 \\
\hline
\end{tabular}

Table 9 Comparison of VDS with other algorithms

To provide further comparisons, the hybrid tabu search of Burke et al. [12, 16] was implemented and tested on the benchmark data sets. The best version of their tabu search (TS2) was applied five times to each instance. Table 10 contains the best and average results. The average execution time on each instance was also recorded. The variable depth search was then set a maximum run time identical to that used by the tabu search for each instance. Five repeats of the variable depth search were then performed to obtain average and best results. Heuristics TR+PG+WD were used.

\begin{tabular}{l|rrr|rrr|r}
\hline & \multicolumn{3}{|c|}{ Variable depth search } & \multicolumn{3}{c|}{ TS2 [12, 16] } & \\
\hline Instance & Best & Average & Avg. Evals. & Best & Average & Avg. Evals. & Time (secs) \\
\hline ORTEC01 & 480 & 1120 & $1,852,788$ & 1581 & 3201 & $2,363,828$ & 108 \\
BCV-1.8.1 & 262 & 269 & 159,379 & 293 & 350 & 140,690 & 9 \\
BCV-2.46.1 & 1574 & 1593 & $1,563,865$ & 1573 & 1596 & $1,557,905$ & 167 \\
BCV-3.46.1 & 3334 & 3346 & $4,486,399$ & 3410 & 3453 & $5,088,206$ & 427 \\
BCV-4.13.1 & 10 & 11 & 152,182 & 11 & 25 & 150,639 & 9 \\
BCV-5.4.1 & 48 & 48 & 21,208 & 48 & 48 & 17,580 & 1 \\
BCV-6.13.1 & 769 & 817 & 356,891 & 1010 & 1154 & 345,595 & 24 \\
BCV-7.10.1 & 381 & 427 & 117,224 & 391 & 458 & 93,817 & 7 \\
BCV-8.13.1 & 148 & 148 & 248,927 & 148 & 165 & 215,524 & 15 \\
BCV-A.12.1 & 1835 & 1942 & 649,926 & 2065 & 2831 & 718,354 & 108 \\
\hline
\end{tabular}

Table 10 Comparison of the variable depth search with a hybrid tabu search 
The results in Table 9 and Table 10 show the variable depth search nearly always outperforms or is equal to previous methods in comparable tests over all instances. The only time it was beaten was when TS2 found a best solution for BCV-2.46.1 with penalty 1573 . The variable depth search could still manage a best with penalty 1574 though. Note that the variable depth search is actually dynamically adjusting to the run time of the other approaches.

\subsection{Longer Computation Times}

Further experiments were conducted to examine the potential benefit of a longer execution time. The variable depth search with the same parameters as before and using heuristics TR+PG+WD was tested on each instance with a time limit of 1 hour. ILS was also tested for comparative purposes.

\begin{tabular}{l|rc|rc}
\hline & \multicolumn{2}{|c|}{ TR+PG+WD } & \multicolumn{2}{c}{ ILS } \\
& Pen & Evals & Pen & Evals \\
\hline ORTEC01 & 435 & $58,703,568$ & 630 & $69,151,556$ \\
BCV-1.8.1 & 254 & $62,769,622$ & 255 & $72,216,494$ \\
BCV-2.46.1 & 1574 & $29,767,138$ & 1594 & $39,255,535$ \\
BCV-3.46.1 & 3302 & $36,422,606$ & 3414 & $42,504,506$ \\
BCV-4.13.1 & 10 & $58,613,414$ & 10 & $68,301,984$ \\
BCV-5.4.1 & 48 & $73,021,195$ & 48 & $92,071,328$ \\
BCV-6.13.1 & 768 & $54,683,305$ & 814 & $62,509,316$ \\
BCV-7.10.1 & 381 & $59,704,319$ & 381 & $69,957,265$ \\
BCV-8.13.1 & 148 & $59,084,678$ & 148 & $66,960,140$ \\
BCV-A.12.1 & 1564 & $23,675,881$ & 1808 & $25,506,048$ \\
Average & 848 & & 910 & \\
\hline
\end{tabular}

Table 11 Experiments with VDS using longer computation times

The increase in computation time leads to an improvement for both methods. Again the simpler ILS examines more solutions in the allotted time but is still not as effective as the variable depth search. In fact, ILS after one hour on each instance is, on average, still worse than the best of five, one minute repeats of the variable depth search with heuristics PG + WD.

\section{Conclusions}

This paper has reviewed search neighbourhoods that have been previously used to solve nurse rostering problems. They were tested using benchmark nurse rostering problems and based on the results, a variable depth search was created.

The block neighbourhood is very effective for the majority of the instances. Today's technology allows these larger neighbourhoods to be exhaustively searched very quickly. Even a simple hill climber which uses these neighbourhoods will produce satisfactory rosters and combining the hill climber with the greedy construction, restart method further improves the quality of solutions produced.

However, the variable depth search can still improve upon this basic iterated local search. The variable depth search works by chaining together the block moves using a number of heuristics to select the next move (link in the chain). The results show the best combination of heuristics to use is PG (positive gain criterion) with TR (time restriction heuristic) and WD (selecting moves on days that have violations which occurred after the last move). 
It is also worth noting that although the variable depth search is more effective, it is also more complicated to implement with an increased potential for introducing errors.

\section{Suggestions for Future Work}

It is possible that the iterated local search can be improved by using more sophisticated heuristics for introducing diversification than the greedy re-construction method. The hill climber using the block neighbourhoods or the variable depth search could also be used in a population based approach such as a memetic algorithm [30] or a scatter search [25] as the means for introducing diversification.

Specific neighbourhoods (i.e. maximum block size settings) and heuristics are particularly effective on certain instances. A method which can exploit this by, for example, intelligently selecting neighbourhoods and heuristics, may be able to contribute gains in performance. One possibility may be an algorithm which runs some short preliminary tests on the instance, testing different parameters and heuristics in order to estimate a good set of parameters for the variable depth search. An alternative approach may be to dynamically adjust the algorithm's set of heuristics and parameters as the search progresses. Somewhat akin to hyperheuristics $[18,43]$. We also plan to create more data sets taken from real world rostering scenarios which along with any new best known solutions will be published on the research website.

\section{Acknowledgements}

This work was supported by EPSRC grant GR/S31150/01.

\section{References}

1. Ahuja, R.K., Ö. Ergun, J.B. Orlin, and A.P. Punnen, A survey of very largescale neighborhood search techniques. Discrete Applied Mathematics, 2002. 123(1-3): pp. 75-102.

2. Aickelin, U., E.K. Burke, and J. Li, An Estimation of Distribution Algorithm with Intelligent Local Search for Rule-based Nurse Rostering. Journal of the Operational Research Society, 2007(in print).

3. Arthur, J.L. and A. Ravindran, A multiple objective nurse scheduling model. AIIE Transactions, 1981. 13(1): pp. 55-60.

4. Azaiez, M.N. and S.S. Al Sharif, A 0-1 goal programming model for nurse scheduling. Computers and Operations Research, 2005. 32(3): pp. 491 - 507.

5. Bard, J.F. and H.W. Purnomo, Preference scheduling for nurses using column generation. European Journal of Operational Research, 2005. 164(2): pp. 510534.

6. Bard, J.F. and H.W. Purnomo, Cyclic Preference Scheduling of Nurses Using A Lagrangian-Based Heuristic. Journal of Scheduling, 2007. 10(1): pp. 5-23.

7. Beddoe, G.R. and S. Petrovic, Selecting and Weighting Features Using a Genetic Algorithm in a Case-Based Reasoning Approach to Personnel Rostering. European Journal of Operational Research, 2006. 175(2): pp. 649671.

8. Beddoe, G.R. and S. Petrovic, Enhancing Case-Based Reasoning for Personnel Rostering with Selected Tabu Search Concepts. Journal of the Operational Research Society, To appear. 
9. Bellanti, F., G. Carello, F.D. Croce, and R. Tadei, A greedy-based neighborhood search approach to a nurse rostering problem. European Journal of Operational Research, 2004. 153: pp. 28-40.

10. Berrada, I., J.A. Ferland, and P. Michelon, A multi-objective approach to nurse scheduling with both hard and soft constraints. Socio-Economic Planning Sciences, 1996. 30(3): pp. 183-193.

11. Brucker, P., E.K. Burke, T. Curtois, R. Qu, and G. Vanden Berghe, Adaptive Construction of Nurse Schedules: A Shift Sequence Based Approach and New Benchmarks. Under review, 2006.

12. Burke, E.K., P. Cowling, P. De Causmaecker, and G. Vanden Berghe, A Memetic Approach to the Nurse Rostering Problem. Applied Intelligence, 2001. 15(3): pp. 199-214.

13. Burke, E.K., T. Curtois, G. Post, R. Qu, and B. Veltman, A Hybrid Heuristic Ordering and Variable Neighbourhood Search for the Nurse Rostering Problem. European Journal of Operational Research, Accepted for publication, to Appear 2007.

14. Burke, E.K., T. Curtois, G. Post, R. Qu, and B. Veltman, A Hybrid Heuristic Ordering and Variable Neighbourhood Search for the Nurse Rostering Problem. European Journal of Operational Research, To Appear.

15. Burke, E.K., P. De Causmaecker, S. Petrovic, and G. Vanden Berghe, Variable Neighborhood Search for Nurse Rostering Problems, in Metaheuristics: Computer Decision-Making, M.G.C. Resende and J.P. de Sousa, Editors. 2004, Kluwer. pp. 153-172.

16. Burke, E.K., P. De Causmaecker, and G. Vanden Berghe. A Hybrid Tabu Search Algorithm for the Nurse Rostering Problem, in Simulated Evolution and Learning, Selected Papers from the 2nd Asia-Pacific Conference on Simulated Evolution and Learning, SEAL 98, Springer Lecture Notes in Artificial Intelligence Volume 1585. B. McKay, et al., Editors. 1999: Springer. pp. 187-194.

17. Burke, E.K., P. De Causmaecker, G. Vanden Berghe, and H. Van Landeghem, The State of the Art of Nurse Rostering. Journal of Scheduling, 2004. 7(6): pp. $441-499$.

18. Burke, E.K., G. Kendall, and E. Soubeiga, A Tabu-Search Hyperheuristic for Timetabling and Rostering. Journal of Heuristics, 2003. 9(6): pp. 451 - 470.

19. Chiarandini, M., A. Schaerf, and F. Tiozzo. Solving Employee Timetabling Problems with Flexible Workload using Tabu Search, in Proceedings of the 3rd International Conference on the Practice and Theory of Automated Timetabling (PATAT-2000). E.K. Burke and W. Erben, Editors. 2000. Konstanz, Germany. pp. 298-302.

20. Darmoni, S.J., A. Fajner, N. Mahé, A. Leforestier, M. Vondracek, O. Stelian, and M. Baldenweck, Horoplan: computer-assisted nurse scheduling using constraint-based programming Journal of the Society for Health Systems, 1995. 5: pp. 41-54.

21. Dias, T.M., D.F. Ferber, C.C. de Souza, and A.V. Moura, Constructing nurse schedules at large hospitals. International Transactions in Operational Research, 2003. 10(3): pp. 245-265.

22. Dowsland, K.A., Nurse scheduling with tabu search and strategic oscillation. European Journal of Operational Research, 1998. 106(2): pp. 393-407. 
23. Ernst, A.T., H. Jiang, M. Krishnamoorthy, B. Owens, and D. Sier, An Annotated Bibliography of Personnel Scheduling and Rostering. Annals of Operations Research, 2004. 127: pp. 21-144.

24. Glover, F., Ejection Chains, Reference Structures and Alternating Path Methods for Traveling Salesman Problems. Discrete Applied Mathematics, 1996. 65(1-3): pp. 223-253.

25. Glover, F., M. Laguna, and R. Martí, Fundamentals of scatter search and path relinking. Control and Cybernetics, 2000. 29(3): pp. 653-684.

26. Jan, A., M. Yamamoto, and A. Ohuchi. Evolutionary Algorithms for Nurse Scheduling Problem, in Proceedings of the 2000 Congress on Evolutionary Computation. 2000. California, USA: IEEE Press. pp. 196-203.

27. Jaszkiewicz, A., A metaheuristic approach to multiple objective nurse scheduling. Foundations of Computing and Decision Sciences, 1997. 22(3): pp. 169-183.

28. Jaumard, B., F. Semet, and T. Vovor, A Generalized Linear Programming Model for Nurse Scheduling. European Journal of Operational Research 1998. 107(1): pp. 1-18.

29. Kostreva, M.M. and K.S.B. Jennings, Nurse scheduling on a microcomputer. Computers and Operations Research, 1991. 18(9): pp. 731-739.

30. Krasnogor, N. and J. Smith, A Tutorial for Competent Memetic Algorithms: Model, Taxonomy and Design Issues. IEEE Transactions on Evolutionary Computation, 2005. 9(5): pp. 474-488.

31. Li, H., A. Lim, and B. Rodrigues. A Hybrid AI Approach for Nurse Rostering Problem, in Proceedings of the 2003 ACM symposium on Applied computing. 2003. pp. 730-735.

32. Lin, S. and B.W. Kernighan, An Effective Heuristic Algorithm for the Traveling-Salesman Problem. Operations Research, 1973. 21(2): pp. 498-516.

33. Lourenço, H.R., O.C. Martin, and T. Stützle, Iterated Local Search, in Handbook of Metaheuristics, F. Glover and G. Kochenberger, Editors. 2003, Kluwer. pp. 321-353.

34. Louw, M.J., I. Nieuwoudt, and J.H. van Vuuren, Finding Good Nursing Duty Schedules: A Case Study.

35. Mason, A.J. and M.C. Smith. A Nested Column Generator for solving Rostering Problems with Integer Programming, in International Conference on Optimisation: Techniques and Applications. L. Caccetta, et al., Editors. 1998. Perth, Australia. pp. 827-834.

36. Meisels, A., E. Gudes, and G. Solotorevsky, Employee Timetabling, Constraint Networks and Knowledge-Based Rules: A Mixed Approach, in Selected papers from the First International Conference on Practice and Theory of Automated Timetabling, Springer Lecture Notes in Computer Science Volume 1154, E. Burke and P. Ross, Editors. 1995. pp. 93-105.

37. Meisels, A. and A. Schaerf, Modelling and solving employee timetabling problems. Annals of Mathematics and Artificial Intelligence, 2003. 39: pp. 4159.

38. Meyer auf'm Hofe, H., Solving Rostering Tasks as Constraint Optimization, in Selected papers from the Third International Conference on Practice and Theory of Automated Timetabling, Springer Lecture Notes in Computer Science Volume 2079 E. Burke and W. Erben, Editors. 2000. pp. 191-212.

39. Miller, H.E., W.P. Pierskalla, and G.I. Rath, Nurse Scheduling Using Mathematical Programming. Operations Research, 1976. 24(5): pp. 857-870. 
40. Nonobe, K. and T. Ibaraki, A tabu search approach to the constraint satisfaction problem as a general problem solver. European Journal of Operational Research, 1998. 106(2-3): pp. 599-623.

41. Petrovic, S., G.R. Beddoe, and G. Vanden Berghe. Storing and adapting repair experiences in employee rostering, in Selected Papers from the 4th International Conference on the Practice and Theory of Automated Timetabling (PATAT 2002), Springer Lecture Notes in Computer Science Volume 2740. E.K. Burke and P. De Causmaecker, Editors. 2003. pp. 149166.

42. Rego, C., A Subpath Ejection Method for the Vehicle Routing Problem. Management Science, 1998. 44(10): pp. 1447-1459.

43. Ross, P., Hyper-heuristics, in Search Methodologies: Introductory Tutorials in Optimization and Decision Support Techniques, E.K. Burke and G. Kendall, Editors. 2005, Springer. pp. 529-556.

44. Thornton, J. and A. Sattar. Nurse Rostering and Integer Programming Revisited, in International Conference on Computational Intelligence and Multimedia Applications. B. Verma and X. Yao, Editors. 1997. pp. 49-58.

45. Valouxis, C. and E. Housos, Hybrid optimization techniques for the workshift and rest assignment of nursing personnel. Artificial Intelligence in Medicine, 2000. 20: pp. 155-175.

46. Vanden Berghe, G. An Advanced Model and Novel Meta-Heuristic Solution Methods to Personnel Scheduling in Healthcare. Ph.D. Thesis, University of Gent, Belgium, 2002.

47. Warner, D.M., Scheduling Nursing Personnel according to Nursing Preference: A Mathematical Programming Approach Operations Research, 1976. 24: pp. 842-856.

48. Yagiura, M., T. Yamaguchi, and T. Ibaraki, A Variable Depth Search Algorithm for the Generalized Assignment Problem, in Meta-Heuristics: Advances and Trends in Local Search Paradigms for Optimization, S. Voss, et al., Editors. 1999, Kluwer Academic Publishers: Boston, MA. pp. 459-471. 